\section{A study of heart failure in the community setting}

Researchers from the Mayo Clinic and Foundation performed a prospective study of 556 patients with heart failure (HF) from Olmsted County, MN, in order to characterize the prevalence and nature of $\mathrm{HF}$ in a community setting.

Over $50 \%$ of the patients had preserved $(\geq 50 \%)$ left ventricular ejection fraction (EF), which was associated with older age, being female, and having no history of myocardial infarction $(P<0.001$ for all). Eighty per cent of the patients had diastolic dysfunction on Doppler imaging; 37\% had combined systolic and diastolic dysfunction, and $44 \%$ isolated diastolic dysfunction. In another study of elderly patients in Olmsted County with cardiovascular disease but no HF, the prevalence of moderate to severe diastolic dysfunction was reported at $17 \%$; in the present study, patients with preserved EF had a $71 \%$ prevalence of moderate or severe diastolic dysfunction (vs $78 \%$ in patients with reduced $\mathrm{EF}$ ). Bursi et al. regard these data as compelling evidence that diastolic dysfunction is vital in the pathophysiology of HF with preserved EF. Of further interest was the fact that reduced $\mathrm{EF}$ and diastolic dysfunction were each independently associated with high plasma levels of brain natriuretic peptide, supporting the use of this biomarker for identifying patients with HF. Interestingly, associated mortality for preserved and reduced EF was similarly high at 6 months, but the authors highlight this as an area for further study.

Original article Bursi F et al. (2006) Systolic and diastolic heart failure in the community. JAMA 296: 2209-2216

\section{Minimizing door-to-balloon time in patients with STEMI}

Prompt treatment of patients with ST-segment elevation myocardial infarction (STEMI) can increase the probability of survival. A crosssectional survey of acute-care hospitals in the US has identified strategies that minimized the interval between arrival at hospital and initiation of reperfusion therapy ('door-to-balloon time') in patients with STEMI who underwent percutaneous coronary intervention.
Bradley et al. received 365 replies from the 500 hospitals surveyed to identify the processes used to treat patients with STEMI. The median door-to-balloon time was $100.4 \mathrm{~min}$, considerably longer than the guideline time of $90 \mathrm{~min}$. Six strategies were found to be particularly effective in minimizing the time to treatment: activation of the catheterization laboratory by the emergency department without authorization by a cardiologist; a single call from the emergency department to a central operator, who pages both the interventional cardiologist and the catheterization laboratory staff; the use of transmitted electrocardiographic data to determine whether the catheterization laboratory should be activated while the patient is en route; minimizing the time between paging and the arrival of staff in the catheterization laboratory; the permanent presence of an attending cardiologist; and the provision of real-time data feedback on doorto-balloon time to the emergency department and catheterization laboratory staff. Hospitals that used four of these strategies had a median door-to-balloon time of $79 \mathrm{~min}$, compared with $110 \mathrm{~min}$ for those that used none.

The authors recommend the implementation of these strategies, particularly those not requiring extra resources, to improve treatment of patients with STEMI.

Original article Bradley EH et al. (2006) Strategies for reducing the door-to-balloon time in acute myocardial infarction. N Engl J Med 355: 2308-2320

\section{Long-term clopidogrel therapy improves outcomes after drug-eluting stent placement}

Patients who receive coronary stents exhibit reduced rates of thrombosis if they receive antiplatelet therapy with thienopyridines, such as clopidogrel, following implantation. FDAapproved indications currently recommend that patients who undergo percutaneous intervention with drug-eluting stents (DESs) should be treated with clopidogrel for 3-6 months; however, evidence indicates that they might be at risk of late-stent thrombosis after discontinuing the drug. In an observational study, Eisenstein et al. have investigated the association between long-term clopidogrel use, and death and myocardial infarction (MI), after DES or bare metal stent (BMS) placement. 